\title{
Sobre la desprotección social
}

\section{José Ignacio del Pozo}

Hurkoa

<jidelpozo@hurkoa.eus>

Artikulu honetan aztertzen dira babesgabetasun sozialeko egoerak gertatzen diren ondorengo hiru kolektibo hauek: pertsona adinduak, hauskortasun egoeran dauden tutoreak eta buruko gaitzak dituzten pertsonak. Hori gauzatzeko, lehenik aztertzen dira kolektibo horietako bakoitzak egunero bizi dituen arrisku-faktoreak zeintzuk diren, eta horren ondoren adierazten dira biztanleriaren talde horiekiko isolamendu eta gizarte-bazterketak dituen berariazko kausa eta ondorioak. Azkenik, bertan nabarmentzen dira babesgabetasunaren alertarako seinale batzuek, eta iradokitzen dira zantzu batzuek politika eta gizarte-zerbitzuen arlotik prebentzioa burutu eta fenomenoa hobe ekiditeko.

\section{GAKO-HITZAK:}

Pertsona adinduak, ezgaitasuna, mendekotasuna, buruko gaitzak, arrisku-faktoreak, gizartebazterketa, babesa.
Este artículo explora las situaciones de desprotección social que se dan en tres colectivos: las personas mayores, las personas tutoras en fragilidad y las personas con enfermedad mental. Para ello, en primer lugar analiza los factores de riesgo a que están expuestos cada uno de ellos, para después esbozar las causas y consecuencias específicas que el aislamiento y la exclusión social tienen en estos grupos poblacionales. Finalmente, identifica algunas de las señales que alertan de una posible desprotección y sugiere pautas para que las políticas y los servicios sociales prevengan y atajen mejor este fenómeno.

\section{PALABRAS Clave:}

Personas mayores, discapacidad, dependencia, enfermedades mentales, factor de riesgo, exclusión social, tutela. 


\section{Introducción}

Referirse a la desprotección social no es fácil. Se trata de un término cuya definición es compleja y sobre al que apenas existe literatura. La referencia principal está relacionada con la protección de los derechos personales, más que con las situaciones de desprotección, por lo que se elude precisamente la definición del término. La legislación vigente plantea las bases para el ejercicio de los derechos de protección de las personas. El inadecuado desarrollo de los deberes de protección establecidos al efecto evidencia las situaciones de desprotección, privando a los individuos de los instrumentos que les permitan gozar de esa protección para un adecuado desarrollo personal y social. No obstante, si se pretende enfocar el análisis social hacia un objetivo concreto, resulta conveniente intentar definirlo.

Realizando una aproximación al concepto, quienes lo han trabajado lo caracterizan como un fenómeno multicausal, progresivo y cambiante. Así, definen la exclusión como:

la imposibilidad o incapacidad de ejercer los derechos sociales. Fundamentalmente el derecho al trabajo, pero también el derecho a la educación, a la cultura, a la salud, a una vivienda digna, a la protección social. No se trata sólo de pobreza o de falta de un hogar, sino también de situaciones de desprotección y exclusión caracterizadas por la ausencia de trabajo y por el aislamiento social. Se trata, normalmente, de situaciones provocadas por una 'acumulación de carencias', de desventajas generalizadas y que, con frecuencia, se mantienen en el tiempo, imposibilitando el ejercicio de los derechos sociales que corresponden a una ciudadanía plena” (Institución de la Procuradora General del Principado de Asturias, 2008: 83).

Una vez aclarado el término, se debe especificar también el lugar en el que nos situamos, los colectivos que atendemos y nuestra pequeña experiencia en la elaboración de proyectos, esto es, el lugar desde el que observamos este fenómeno social. La experiencia de Hurkoa se refiere a la atención de personas mayores, incapacitadas o no, a personas con problemas cognitivos y de salud mental, y, cuando existen, a las personas que ostentan su tutela. Al objeto de continuar ampliando la atención prestada, a partir de 2014 se ha realizado un estudio sobre las condiciones de vida y las necesidades de las personas en situación de fragilidad -en referencia principal a las mayores de 64 años-, sus características, condiciones de vida y necesidades.

Cuando se habla de desprotección, el término se relaciona con las diferentes situaciones de maltrato, que en función del modo o los tipos de conducta, se pueden producir, como son el abuso intencionado hacia terceras personas, la negligencia y el abandono, el desamparo, el aislamiento o la vulnerabilidad, entre otras. Todas esas manifestaciones de desprotección terminan afectando seriamente a distintas esferas de la persona y pueden darse en distintos ámbitos, tanto en el de los cuidados personales como en el psicológico y el económico.

El trabajo de Hurkoa se ha centrado históricamente en la atención a personas mayores o con enfermedad mental, y es por ello por lo que en este artículo queremos centrarnos en estos colectivos, por ser los que mejor conocemos. Desde nuestro enfoque particular, el perfil de persona en situación de fragilidad es el de una mujer que vive sola (o convive con otras personas de su misma edad o mayores), con poca red social o con poca efectividad de ésta. A estas circunstancias de partida, se pueden sumar algunos condicionantes que pueden agravarla o complejizarla, como es el hecho de tener una economía precaria que puede derivar en la 'pobreza vergonzante', que hace que la persona en cuestión no demande ni aquellas ayudas a las que legalmente tiene derecho (por ejemplo, el complemento de pensión o la renta de garantía de ingresos). Otro condicionante es la soledad: en una sociedad como la nuestra, en la que la familia y las relaciones de amistad tienen una consideración social positiva, su carencia o ineficacia puede llevar a la persona a sentirse responsable de esa situación. Asimismo, las barreras arquitectónicas del domicilio y del barrio no facilitan el mantenimiento de relaciones sociales normalizadas, abocando a un progresivo aislamiento que puede llegar a culpabilizar al individuo en soledad, o que puede provocar un progresivo proceso de 'acomodación'. Con independencia de las causas que la provoquen, esta soledad culpabilizante 0 'acomodada' induce al individuo a no demandar.

En el estudio que estamos realizando sobre las personas mayores en situación de fragilidad, se ha hecho evidente que ésta es una condición que permea todas las edades de la vida. Así, se consideran personas en situación de fragilidad quienes en la infancia sufrieron algún tipo de maltrato, cuyas carencias y consecuencias afloran en la edad adulta; las criaturas con progenitores negligentes; los jóvenes, emigrantes o no, que viviendo en pisos de acogida, llegan a la mayoría de edad y, de un día para otro, se ven despojados de los recursos de que hasta ese momento habían disfrutado; las personas emigrantes en exclusión o desarraigo, máxime los 'sin papeles', es decir, las personas en situación de ilegalidad; las personas sin ingresos o con rentas de trabajo insuficientes para asegurar su supervivencia y la de los suyos ${ }^{1}$; y cómo no, las personas con enfermedad mental, con independencia de su edad, las personas con déficits cognitivos, las personas 'tutoras en fragilidad' $2 \mathrm{y}$, por

${ }^{1}$ Éste es el caso de las parejas con descendencia y con trabajos precarios o que están en paro, y que viven gracias a las ayudas provenientes de sus progenitores ya jubilados, lo que en demasiadas ocasiones supone repartir la precariedad económica.

${ }_{2}$ Término acuñado por Hurkoa para designar de forma explícita a personas mayores que tienen a su cargo - por mandato legal- a 
supuesto, quienes se encuentran en una situación de soledad no elegida, con independencia de su edad y condición. Cabe apuntar que todas estas situaciones tienen una dificultad añadida cuando se producen en el medio rural. La idiosincrasia del medio, la mayor dificultad para acceder a los recursos sociales, unida a una mayor reticencia a demandar ayudas externas pueden suponer un plus de riesgo.

Con anterioridad se ha dicho que vamos a centrar nuestro análisis en los colectivos que mejor conocemos, esto es, las personas mayores, las personas tutoras en fragilidad y las personas con enfermedad mental. Se deja al margen de nuestro enfoque al colectivo de personas con déficits cognitivos, al entender que, en nuestro contexto, cuentan con estructuras de apoyo orientadas a la salvaguarda de sus derechos ciudadanos.

\section{Las personas mayores}

En los últimos años se ha hecho evidente el papel que factores como la exclusión social, el aislamiento y la soledad juegan en el desarrollo vital de las personas, en su conjunto, y de las personas mayores, en particular. De acuerdo con los datos del Eustat (2014), en Euskadi las personas mayores de 65 años suponen casi el $21 \%$ del total de la población. En el estudio que estamos realizando sobre fragilidad, y concretamente al analizar los datos referentes a la ciudad de Irun, en la que se está procediendo a desarrollar el plan piloto del estudio, las personas mayores de 68 años suponen el 17,2\% de la población, y de ellas entre el $23 \%$ y el $24 \%$ viven solas (Ayuntamiento de Irun, 2015). De las que viven solas, el $77 \%$ son mujeres, es decir, que 3 de cada 4 personas mayores que viven solas son mujeres; entre ellas, una parte vive con ingresos insuficientes para poder hacer frente a las necesidades básicas vitales. Sin embargo, a pesar de sus condiciones de soledad y pobreza, una gran parte de ellas se encuentra integrada en la sociedad, goza de una calidad de vida aceptable y tiene apoyos familiares y sociales.

\subsection{Factores de riesgo}

Además de la edad, el propio proceso involutivo que caracteriza a este colectivo es un factor de riesgo, y lo mismo cabe decir de la precariedad económica asociada a recursos insuficientes, que puede desembocar en una pobreza vergonzante. También la ausencia de interlocutores de confianza y la falta de apoyos tanto en la red formal como en la informal -bien por ausencia o por envejecimiento de ésta, bien por la pérdida de su efectividad-aboca al

descendientes con problemas de salud física o mental. Se trata de personas que son conscientes de su envejecimiento, de la disminución de sus capacidades y de la responsabilidad que asumen. Están hondamente preocupadas por el futuro, sobre todo por el de sus tutelados, y para continuar desarrollando esa función de tutela, necesitan ser apoyados y acompañados. colectivo a situaciones de soledad y desamparo preocupantes, que, por desconocimiento de sus derechos o por la dificultad que tienen estas personas para hacer uso de ellos, pueden derivar en situaciones de autoabandono y de negligencia - llevando a que desistan de demandar la atención que legalmente les corresponde- e incrementar el riesgo de sufrir situaciones de abandono e incluso de abuso y maltrato.

Relacionada con el proceso involutivo, la disminución de la capacidad de autocrítica y la pérdida progresiva de conciencia de encontrarse en situación de necesidad implica, en ocasiones, que aunque estas personas se encuentren en situación de necesidad, no demanden.

La precariedad de la vivienda, sumada a los problemas de accesibilidad tanto en ésta como en el entorno en que se sitúa, también limita la capacidad de autogestión y de mantenimiento de las relaciones sociales. Estas situaciones se ven agravadas cuando una persona de edad avanzada asume la responsabilidad del cuidado o la tutela de terceras personas.

Como anteriormente se ha expuesto, el riesgo se agrava cuando nos referimos a personas mayores que viven en el medio rural. La dispersión que lo caracteriza y la mayor lejanía de los recursos complican su situación, máxime cuando se trata de la única generación que vive en el caserío.

\subsection{Consecuencias}

La principal consecuencia es la disminución progresiva de la capacidad de gestión del día a día y el deterioro progresivo de las condiciones de vida, lo que, junto con el aislamiento y la soledad, aboca a estas personas a unas condiciones de vida no dignas.

\subsection{Causas}

El deterioro progresivo físico y psíquico asociado al envejecimiento y la falta de recursos de apoyo y acompañamiento para este colectivo es una de las principales causas de desprotección.

\subsection{Señales de alerta}

Las señales de alerta son, en primer lugar, la demanda directa y, cuando ésta no se produce, la posible detección de la reducción de su actividad cotidiana, de sus relaciones sociales y el aumento de sus dificultades en la gestión de su vida y su economía. Este proceso solamente es posible detectarlo desde la cercanía. De ahí el valor de los servicios sociales y de la red social informal. 


\subsection{Cómo intervenir}

Algunas de las líneas de actuación que pueden desarrollarse para dar una respuesta acorde a las necesidades existentes son:

- Mejorar la atención a las personas mayores mediante programas de prevención y de atención temprana e individualizada, y poner en marcha mecanismos que permitan la identificación de necesidades y el establecimiento de medidas que les den respuesta, para desarrollar una atención adecuada en el domicilio o en la institución.

- Ampliar y mejorar la oferta de viviendas tuteladas y plazas de residencias que hagan más fácil, cuando sea necesario, dejar la vivienda habitual, que ofrezcan mayores garantías y seguridad, adecuadas a las necesidades personales, sin que ello conlleve necesariamente una desubicación.

- Mejorar la información sobre cuestiones legales que faciliten la toma de decisiones y la planificación de la última etapa de la vida conforme a sus deseos y valores.

- Realizar campañas de sensibilización, a fin de que la ciudadanía se convierta en agente activo de apoyo y prevención de las personas mayores en situación de desprotección.

\section{Las personas tutoras en fragilidad}

En este epígrafe nos referiremos a las personas mayores que han asumido la tutela legal de un descendiente, bien por tener déficits cognitivos, enfermedad mental o por otras causas. La situación de estas personas es desconocida para la sociedad en general, si exceptuamos las asociaciones de atención a estos colectivos, que, en su mayoría, han abordado desde hace tiempo los problemas asociados a la tutela. En la medida en que Hurkoa atiende a personas mayores, desde hace algún tiempo venimos detectando la necesidad de atención que este colectivo presenta.

\subsection{Factores de riesgo}

Al igual que las personas mayores y muy mayores, el principal factor de riesgo de las personas tutoras en fragilidad es el envejecimiento, y derivado de éste, la dificultad progresiva para asumir las tareas y, sobre todo, la responsabilidad propia de la tutela. Un factor de riesgo asociado es la precariedad económica y las situaciones de pobreza.

\subsection{Consecuencias}

La consecuencia inmediata es la pérdida de efectividad. Generalmente son personas que han asumido la tutela de un descendiente, entendiéndola como una responsabilidad y un deber moral. La pérdida progresiva de su capacidad para la gestión, tanto de su propia vida como de las obligaciones propias de la tutela, cuando no se cuenta con apoyos externos - bien de la red formal, bien de la informal- para llevar a cabo esta responsabilidad que entienden es suya e intransferible hace que se sientan frágiles, y a la vez, culpables por no poder gestionarla, lo que reduce su capacidad de gestión.

\subsection{Causas}

La causa obvia es la edad y el propio proceso involutivo de la persona tutora y la sobreimplicación, no efectiva, en una tarea que excede a sus capacidades actuales y que ha entendido como responsabilidad propia. La incertidumbre respecto del futuro de la persona tutelada representa una carga moral difícil de soportar. La soledad, la falta de interlocutores, la falta de información y la responsabilidad no compartida hacen más pesada, si cabe, la asunción de una responsabilidad que ya no es posible asumir.

\subsection{Señales de alerta}

Como en otras situaciones de desprotección, la principal señal de alerta es la demanda directa. El desconocimiento de cuestiones legales y la falta de una interlocución válida limitan la capacidad de estas personas para planificar acciones efectivas.

Otra señal que debe ser tenida en cuenta es la dificultad progresiva en las tareas de gestión de la tutela, que se puede ir manifestando en los espacios jurídico, económico y de gestión de la vida cotidiana.

\subsection{Cómo intervenir}

Las intervenciones con este colectivo se deben dirigir hacia el diagnóstico temprano y hacia la atención de sus necesidades, y deben abordar diferentes áreas:

- Acompañamiento y trabajo a tres bandas -con la persona tutora en fragilidad, con la persona tutora de sustitución y con la persona tutelada-, a fin de realizar un acompañamiento efectivo en el traspaso progresivo de la responsabilidad de la tutela y de facilitar la estructuración de un proceso tutelar respetuoso con las creencias y valores desarrollados a lo largo de la tutela. Al mismo tiempo, se ha de realizar un trabajo vincular entre la persona tutelada y la tutora de sustitución, paliando así un proceso más traumático consecuente al fallecimiento de la persona tutora en fragilidad.

- Mejora en la información sobre cuestiones legales que faciliten la toma de decisiones y la planificación de la vida de la persona tutelada, así como de la última etapa de la vida de los y las tutores en fragilidad, conforme a sus deseos y valores. 
- Lo importante en la intervención con estas personas que han venido haciéndose cargo de todas las gestiones de otra es ofrecerles el apoyo necesario en aquellos asuntos que les resulten más complejos, siempre respetando y priorizando lo que ellas mismas demanden. Esto requiere de su total aprobación y colaboración, es decir, supone la necesidad de ir acordando conjuntamente aquellos aspectos de sus vidas y aquellas gestiones en las que se va a intervenir. De esta forma, se van creando vínculos de confianza que, con posterioridad y llegado el momento, facilitarán la labor de la persona que ejercerá como tutora, haciéndose cargo de la otra persona.

\section{Las personas con enfermedad mental}

Las personas con enfermedad mental ${ }^{3}$ tienen, en nuestro contexto, un riesgo añadido de desprotección y de exclusión social, debido a su idiosincrasia, a la escasez de recursos para su atención psicosocial y al hecho de que los criterios utilizados para baremar el nivel de dependencia están mayoritariamente orientados a valorar la dependencia física. Las personas con enfermedad mental no tienen limitaciones funcionales; por el contrario, una de las características de la enfermedad mental es, precisamente, la imposibilidad de hacer uso de las propias capacidades de forma que permitan disfrutar de una autonomía personal.

\subsection{Factores de riesgo}

Los principales factores de riesgo de este colectivo están relacionados con los síntomas, factores y condicionantes propios de la enfermedad que limitan o imposibilitan a la persona para hacer ejercicio cabal de su autonomía. Uno de los síntomas más limitantes es la apatía que acompaña a este tipo de procesos. La apatía como síntoma no es fácil de entender; no obstante, es necesario tenerla muy en cuenta a la hora de planificar el abordaje de la enfermedad mental grave.

Otro factor que se ha de considerar es la edad. La enfermedad mental permea todos los tramos de la vida, desde la infancia a la vejez, y existe una reconocida carencia en los dispositivos de atención a las personas adultas con enfermedad mental, por lo que es necesario plantearse la atención de este colectivo cuando envejece.

Relacionado también con la sintomatología y las características propias de la enfermedad, es necesario hablar de la falta de conciencia de enfermedad que caracteriza a estas personas. Aunque resulte difícil de entender, conviene tener en

${ }^{3}$ Se estima que la enfermedad mental grave (esquizofrenia y otras psicosis) afecta aproximadamente al $1 \%$ de la población. Es a este colectivo al que nos vamos a referir. cuenta que, aun siendo conscientes del sufrimiento que su estado les provoca, no se sienten enfermas y en muchos casos no van a demandar atención psicosocial. No es un colectivo demandante ni reivindicativo; la propia enfermedad limita en gran medida su capacidad de estructurarse como colectivo, o dicho de otro modo, su capacidad para alcanzar una eficacia como colectivo reivindicativo está muy mermada.

Puesto que la enfermedad se manifiesta en lo social, debe señalarse que son personas con las que no resulta fácil convivir y que resultan socialmente incómodas. Por último, el no diagnóstico es también un importante riesgo asociado.

\subsection{Consecuencias}

Las personas con enfermedad mental tienen un mayor riesgo de exclusión social, y por lo tanto, de desprotección. La esquizofrenia afecta al $1 \%$ de la población general; no obstante, el porcentaje de personas con enfermedad mental aumenta considerablemente cuando nos referimos a las personas que viven en la calle, los 'sin techo', grupo en el que alcanza aproximadamente el $15 \% 4$. La enfermedad les limita en su vida y en la gestión de sus problemas de salud mental. Esto provoca un deterioro progresivo de su autonomía hasta llegar a la incapacidad para manejarla, lo que cual, a su vez, deriva en un mayor riesgo de aislamiento y de deterioro físico.

Otra cuestión que es preciso señalar es la dificultad en la adherencia al tratamiento. Si bien ésta es una característica asociada a cualquier enfermedad crónica, y las psicosis lo son, en este caso se dan otros condicionantes que influyen negativamente en el desarrollo de una buena adherencia, como son la falta de conciencia de la enfermedad y los efectos secundarios de la medicación.

Puesto que la enfermedad dificulta también las relaciones con los demás, este colectivo tiene un mayor riesgo de vivir situaciones de soledad, sin olvidar el estigma social que acompaña a la enfermedad mental.

Resumiendo, los condicionantes propios de la enfermedad mental abocan a estas personas a la soledad y la exclusión. El riesgo de desprotección social de este colectivo ha sido reconocido de forma generalizada, y el Comité Económico y Social Europeo (2011) recomienda utilizar una herramienta específica para su estudio: la tipología europea de personas sin hogar (European typology on homelesseness, ETHOS). La falta de recursos para la correcta atención

${ }^{4}$ El II Estudio sobre la situación de las personas en situación de exclusión social grave en la CAPV (SIIS Centro de Documentación y Estudios, 2014), al referirse a la salud en general de este colectivo, apunta a que es peor que la de la población general. Así, el 15,2\% de las personas sin hogar tienen una enfermedad mental y el $37,3 \%$ sufren alguna dolencia grave o crónica. 
psicosocial de este colectivo incide negativamente en sus condiciones de vida y salud, aumentando su riesgo de exclusión y de desprotección.

\subsection{Causas}

Las causas de que el riesgo de exclusión social y de desprotección sea mayor para las personas con enfermedad mental respecto a quienes no la padecen se debe a los propios síntomas de la enfermedad y a la precariedad de los recursos asistenciales existentes. Nadie dudó en su momento de las bondades de lo que se denominó reforma psiquiátrica. Dejar atrás los manicomios, en tanto que instituciones cerradas y con poca capacidad de tratamiento, fue una buena medida. Lo que faltó fue desarrollar los recursos asistenciales que el colectivo necesita para evitar su exclusión. Se cerraron los manicomios y, por desgracia, en nuestro contexto las personas con enfermedad mental fueron 'devueltas', en el mejor de los casos, a sus familias, y en la mayoría, a la calle, donde viven marginadas y desprotegidas.

\subsection{Señales de alerta}

Las señales de alerta no son fáciles de detectar. Al no haber conciencia de enfermedad, la demanda directa no es habitual. La dificultad en la adherencia a los tratamientos o el no acudir a las consultas programadas hace que sean personas que 'se pierden', que desaparecen del sistema sanitario si éste no realiza un seguimiento planificado.

Las dificultades para la convivencia y las relaciones sociales hacen que sea un colectivo con muchas dificultades para integrarse en el mundo laboral y desarrollar una vida laboral continuada. Por desgracia, las señales de alerta se producen tarde, cuando estas personas han roto con sus vínculos afectivos y sociales y llegan a la marginación social.

Un riesgo añadido es el consumo de sustancias, que generalmente 'ayuda' a paliar los síntomas de la enfermedad.

\subsection{Cómo intervenir}

Está claro que es absolutamente necesario y urgente mejorar la atención general a este colectivo, mejorando el diagnóstico y el desarrollo de los dispositivos de atención psicosocial. Asimismo, conviene aumentar la oferta de dispositivos asistenciales de baja intensidad y optimizar el seguimiento permanente e individualizado de los casos. Y, por supuesto, modificar los baremos de evaluación de la discapacidad y dependencia ajustándolos a la idiosincrasia del colectivo, a las características de la enfermedad y sus síntomas, $y$ a las limitaciones que produce en las personas. La enfermedad mental no provoca una afectación funcional, y sí, en cambio, una importante limitación para la gestión y el manejo de la autonomía personal.

\section{Para terminar}

En definitiva, la respuesta a las personas en situación de desprotección y desamparo requiere que el conjunto de las instituciones y servicios desarrollen políticas inclusivas, que pongan en el centro de éstas a las personas en situación de riesgo. Para ello, un requisito imprescindible es la coordinación interinstitucional y la integralidad de las respuestas.

Como líneas de actuación, se proponen las siguientes:

- Definir con claridad el ámbito competencial de cada servicio, pero con una visión colaborativa, no obstruccionista. Fortalecer la coordinación de los diferentes servicios: vivienda, servicios sociales, salud y salud mental. La coordinación no debe descansar exclusivamente en la voluntad de los profesionales, sino que han de establecerse cauces formales de trabajo compartido entre los distintos agentes concernidos.

- Dotar a los servicios y a las personas que en ellos trabajan de la capacidad y las herramientas para coordinarse con todos los recursos disponibles, y poder así realizar el acompañamiento y el seguimiento de la persona en situación de desprotección de forma integral. Aquí sería fundamental disponer de la figura del profesional de referencia, que asuma una función de seguimiento, de acompañamiento de la persona atendida y de coordinación respecto de los diferentes recursos que necesite. 


\section{Bibliografía referenciada}

COMITÉ ECONÓMICO Y SOCIAL EUROPEO (2012):

"Dictamen del Comité Económico y Social Europeo sobre 'El problema de las personas sin hogar", Diario Oficial de la Unión Europea, 2012/C 24/07, 28-1-12, págs. 35-39 [<http://eur-lex.europa.eu/legal-content/ ES/TXT/?uri=CELEX\%3A52011|E1592>]. AYUNTAMIENTO DE IRUN (2015): Padrón municipal.

EUSTAT (2014): Panorama demográfico, Vitoria-Gasteiz, Eustat [<http://www.eustat.eus/elementos/ eleo012200/ti_Panorama_demografico_2014/ info012240_c.pdf〉].
INSTITUCIÓN DE LA PROCURADORA GENERAL DEL PRINCIPADO DE ASTURIAS (2008): $23^{\text {as }}$ Jornadas de Coordinación de Defensorías. Las personas en situación de desprotección social grave. Asturias 2008 [<http://www.ararteko.net/ RecursosWeb/DOCUMENTOS/1/0_1874_1.pdf〉].

SIIS CENTRO DE DOCUMENTACIÓN Y ESTUDIOS (2015): I/ Estudio sobre la situación de las personas en situación de exclusión residencial grave en la CAPV / EAEn bizitegi-bazterketa larrian daudenen egoerari buruzko II. Azterketa, Donostia-San Sebastián, SIIS Centro de Documentacion y Estudios [<http://www.siis. net/es/investigacion/ver-estudio/347/>]. 